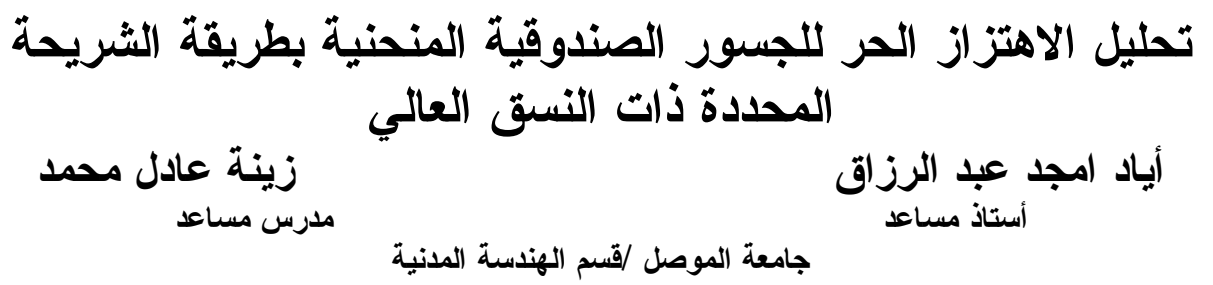

الخلاصة

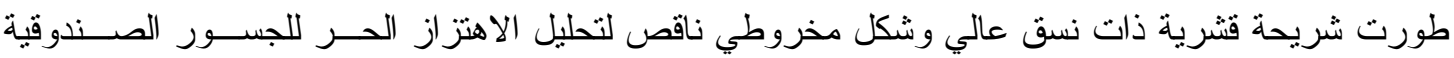

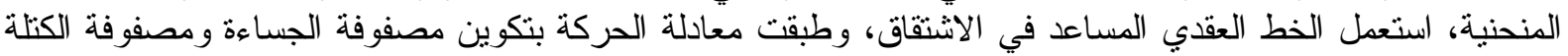

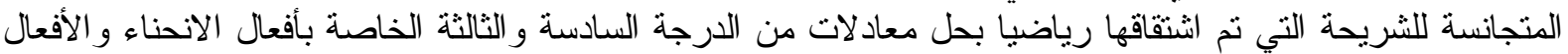

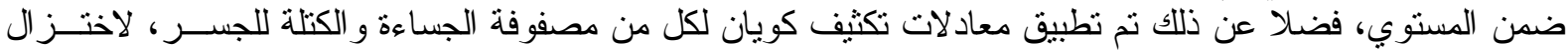

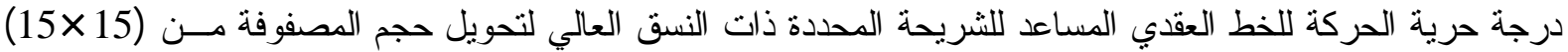
إلى (8) (8)

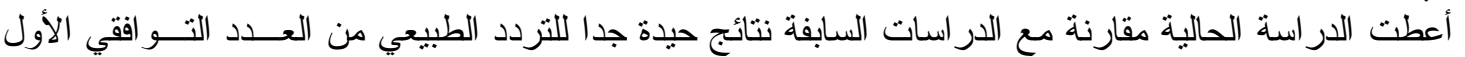

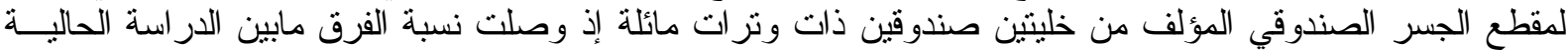
و السابقة إلى (6.73\% )، أما بالنسبة للتردد الطبيعي من العدد التو افقي الثاني لنفس المقطع فقد وصلت نسبة الفرق إلـى لـى

الكلمات الدالة: تحليل الاهتزاز الحر، الجسور الصندوقية المنحنية، الثريحة المحدة ذات النسق العالي.

\title{
Free Vibration Analysis Of Curved Box Girder Bridges by Higher Order Finite Strip
}

\author{
Ayad A. Abdul Razzak \\ Assistant Professor
}

\author{
Zeena A. Mohamma \\ Assistant Lecturer
}

Mosul University/ Civil Engineering Department

\begin{abstract}
A higher-order conical frustum shell strip for analysis of free vibration of curved box girder bridges is developed, the auxiliary nodal line technique is used in the formation, the application of the motion equation by form the matrices of the stiffness and that of the consist mass of the strip and which has been mathematically derived by solving sixth and third order equations for bending and in plane actions respectively. Guyan reduction technique have been applied for each stiffness and the mass matrices of bridge. The strip which implies minimizing the degrees of freedom of the auxiliary nodal line of the Higher Order Finite Strip in order to reduce the size of the matrix from $(15 \times 15)$ to $(8 \times 8)$.

The study has come out with very good results compared to the previous studies in terms of the natural frequencies for the first harmonic number of the box girder bridge section which is composed of two box cells with inclined webs. The ratio of difference between the present and previous studies have reached $(6.73 \%)$, while the natural frequencies for the seconed harmonic number of the same box section the ratio is about equal (3.765\%) .
\end{abstract}

Keywords: Box Girder Bridges, Free Vibration Analysis, Higher Order Finite Strip. 


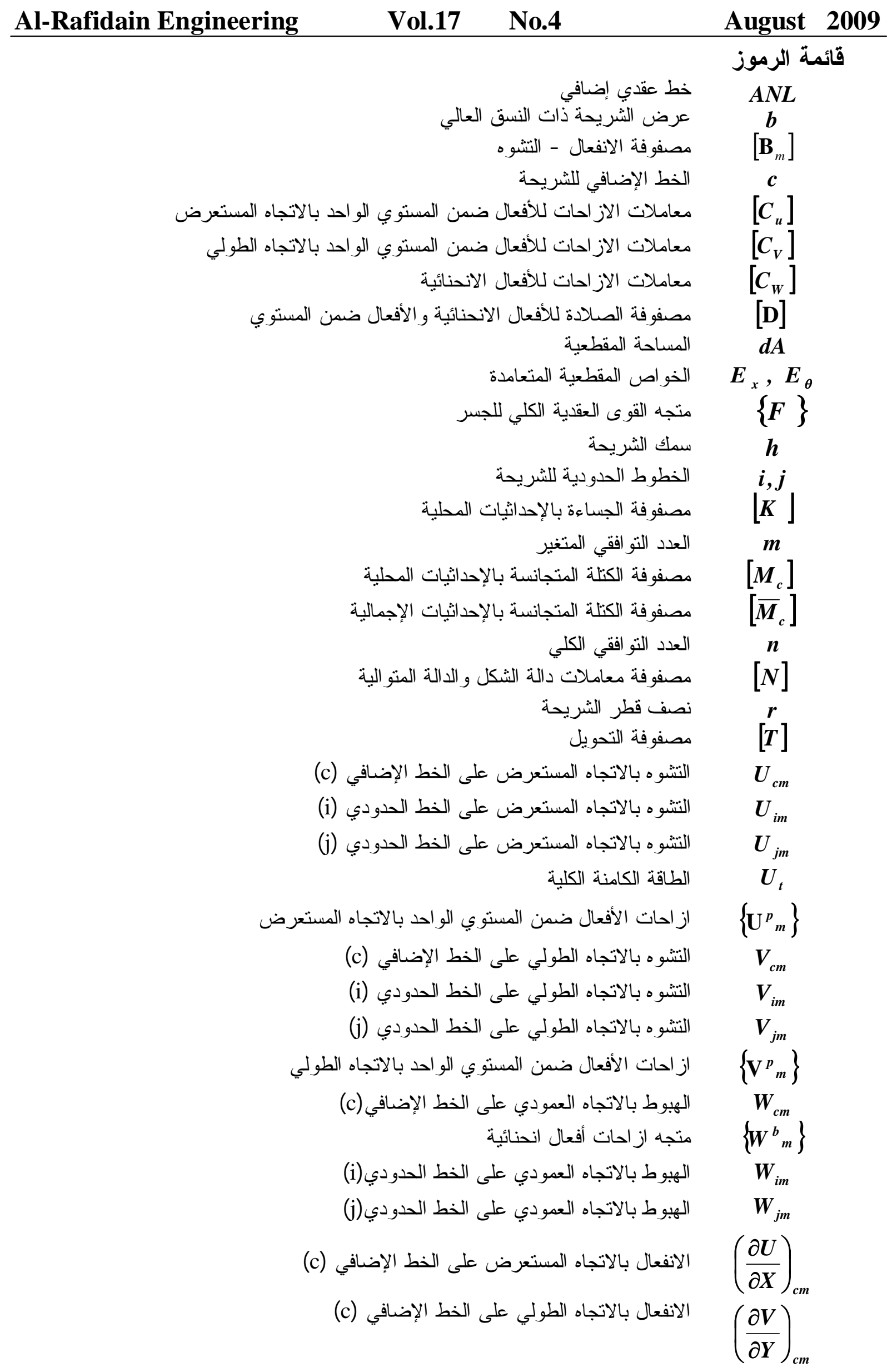




$$
\begin{aligned}
& \text { زاوية انحر اف الجسر المنحني أفقيا }
\end{aligned}
$$

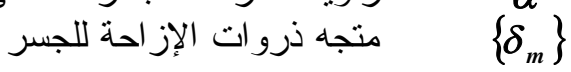

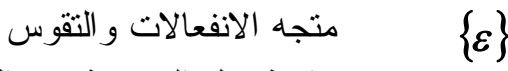

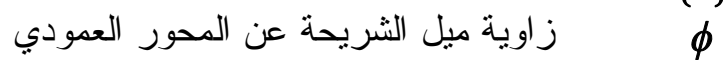

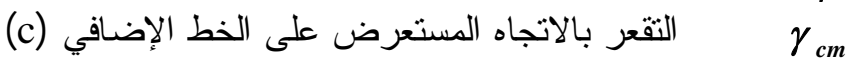

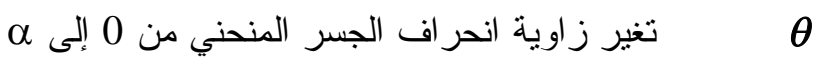

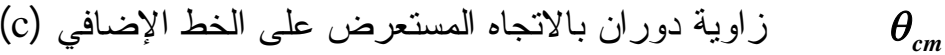

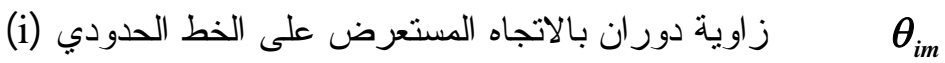

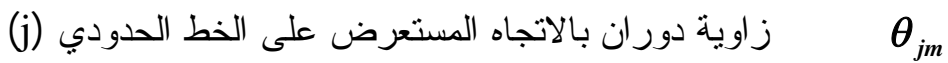

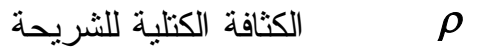

$$
\begin{aligned}
& \text { منجه الاجهادات و العزوم } \quad\left\{\sigma_{m}\right\} \\
& \quad \omega_{1,} \omega_{2}
\end{aligned}
$$

المقدمة

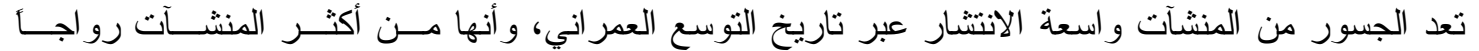

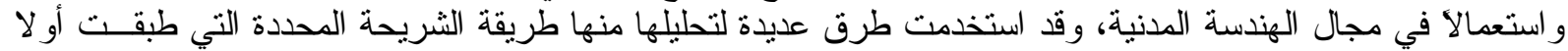

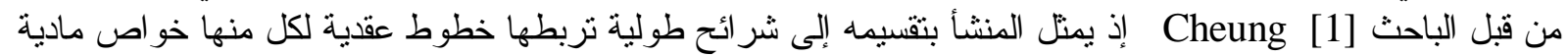

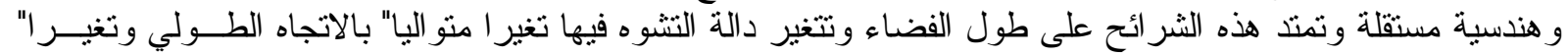
متعدد الحدود بالاتجاه العرضي.

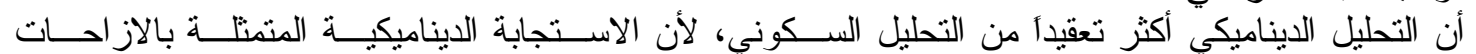

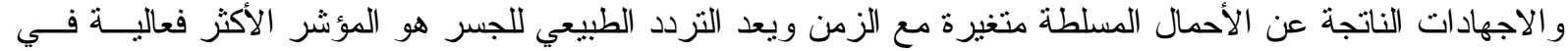

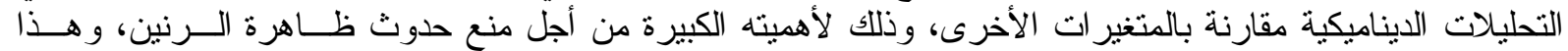

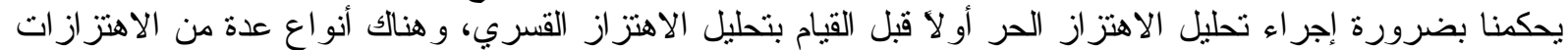

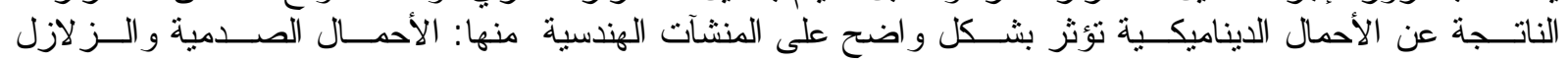

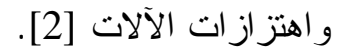

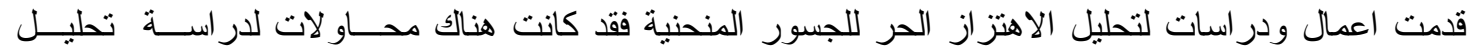

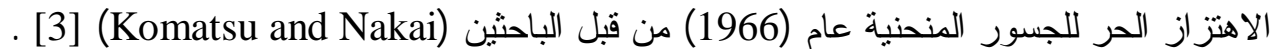

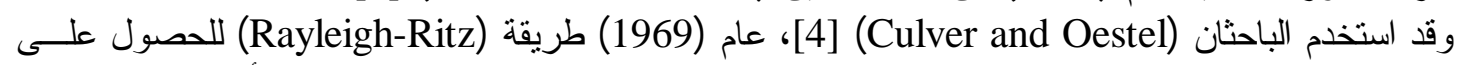

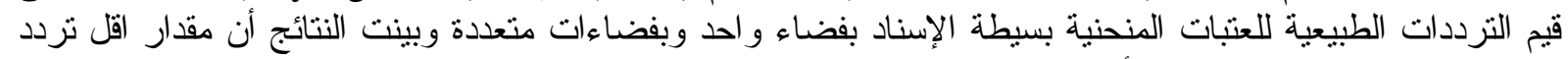

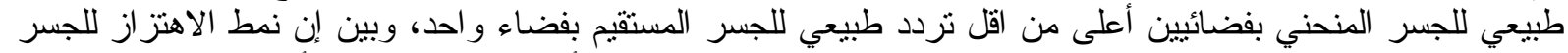

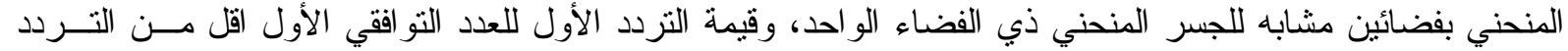

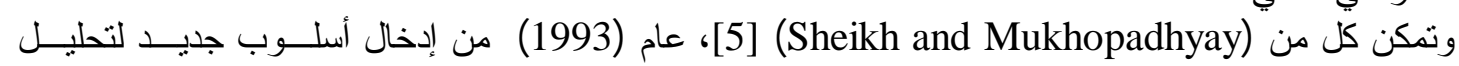
الاهتزاز الحر للمنشآت الصفائحية المقو اة بطريقة(Spline Finite Strip) التي تشتخدم مع نماذج الجسور، إذ أعطت

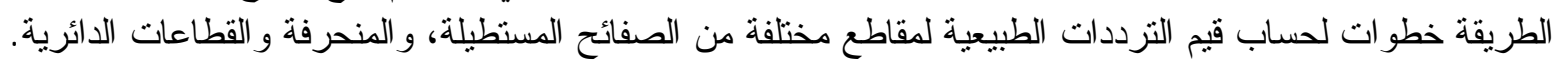

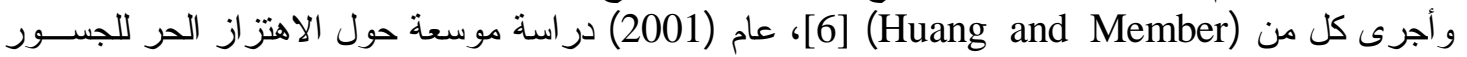

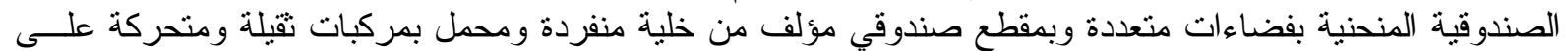

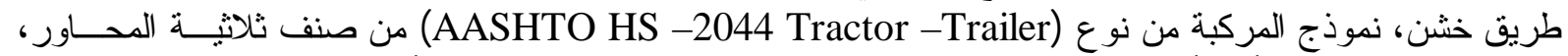

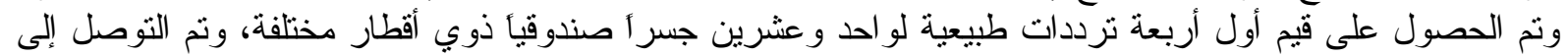

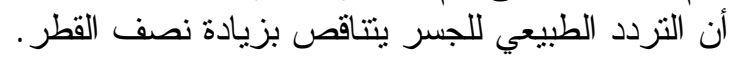

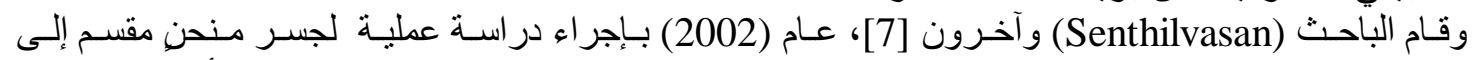

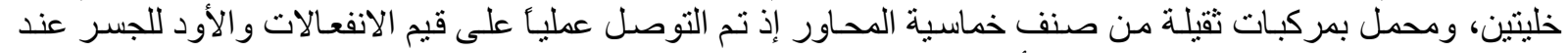

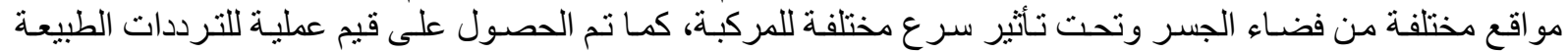


وفي عام (2005) قام الباحثان (Abdul-Razzak and Mohammad) [8)، باســنخدام طريقــة الثــريحة

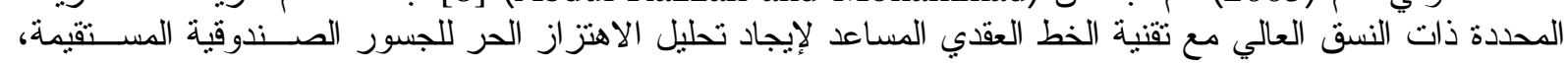

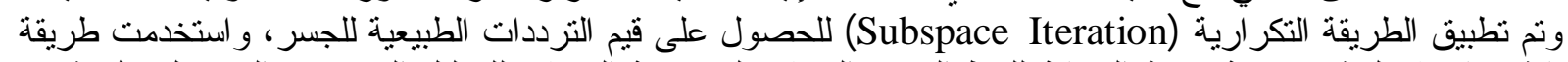

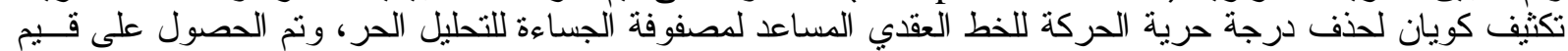
التزددات الطبيعية للعدد التو اقفي الأول.

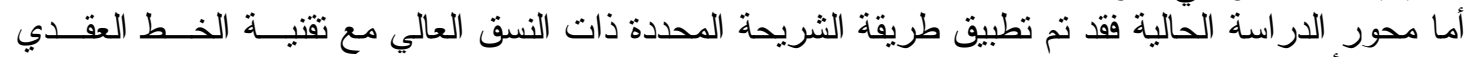

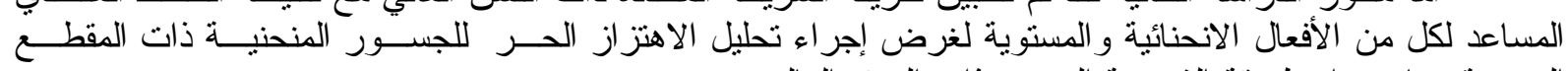
الصندوقي باستخدام طريقة الشريحة المحددة ذات النسق العالي.

\section{الفرضيات الأساسية للشريحة القشرية المنحنية ذات النسق العالي

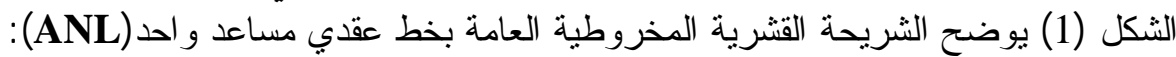

استخدمت دالة الإزاحة من المرتبة السادة لتمثيل التغاير للأفعال الانحنائية (

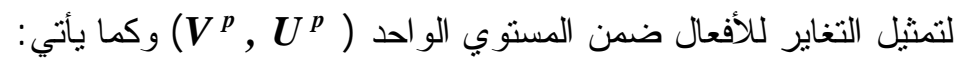

أفعال الاحناء: يمكن التعبير عن دالة الإزاحة للأفعال الانحنائية بالصيغة الآتية [11,10,9] :

$W^{b}=\sum_{m=1}^{n}\left[C_{W}\right]\left\{W^{b}{ }_{m}\right\} \sin \frac{m \pi \theta}{\alpha}$

$=\sum_{m=1}^{n}\left[C_{1}, C_{2}, C_{3}, C_{4}, C_{5}, C_{6}, C_{7}\right]\left\{W^{b}{ }_{m}\right\} \sin \frac{m \pi \theta}{\alpha} W^{b}$

$C_{1}=1-39 S^{2}+162 S^{3}-267 S^{4}+216 S^{5}-64 S^{6}$

$C_{2}=x\left(1-8 S+25 S^{2}-38 S^{3}+28 S^{4}-8 S^{5}\right)$

$\boldsymbol{C}_{3}=48 \boldsymbol{S}^{2}-224 \boldsymbol{S}^{3}+432 \boldsymbol{S}^{4}-384 \boldsymbol{S}^{5}+128 \boldsymbol{S}^{6}$

$C_{4}=x\left(-8 S+32 S^{2}-40 S^{3}+16 S^{4}\right)$

$C_{5}=x^{2}\left(2-12 S+26 S^{2}-24 S^{3}+8 S^{4}\right)$

$C_{6}=-9 S^{2}+62 S^{3}-156 S^{4}+168 S^{5}-64 S^{6}$

$C_{7}=x\left(S-7 S^{2}+18 S^{3}-20 S^{4}+8 S^{5}\right)$

$S=x / b$

$\left\{W^{b}{ }_{m}\right\}=\left[\mathbf{W}_{i m}, \theta_{i m}, \mathbf{W}_{c m}, \theta_{c m}, \gamma_{c m}, \mathbf{W}_{j m}, \theta_{j m}\right]^{r}$

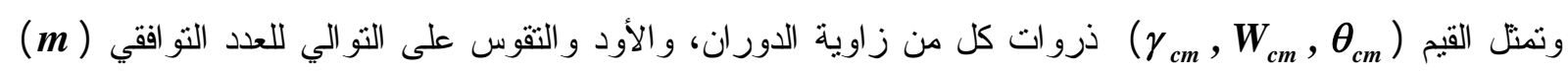
عند الخط العقدي المساعد (ANL). (لف

الأفعال ضمن المستوي الواحد: لتمثيل الإز احة ضمن المستوي الواحد ( الدرجة، الني يمكن التعبير عنها بالمعادلة الآتية [11,10,9]: 

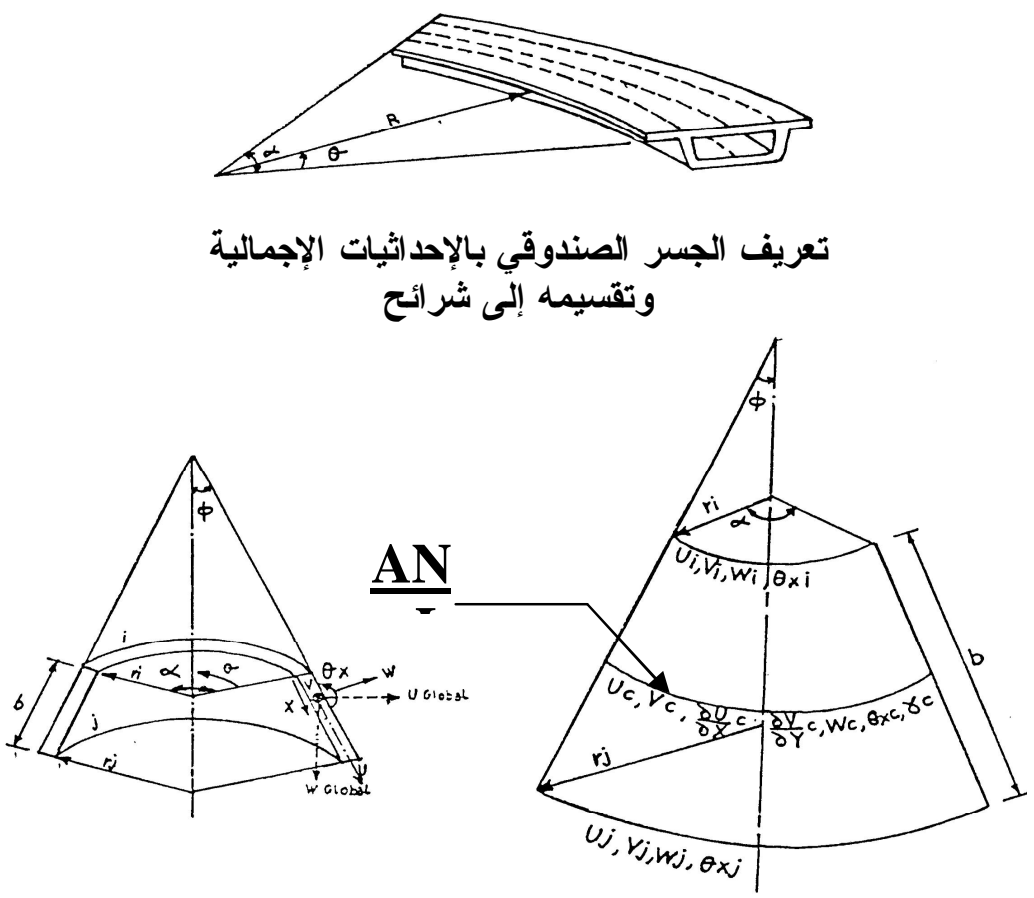

درجة حرية الثريحة ذات النسق العالي إحداثيات الثريحة المخروطية المنحنية المستخدمة في التحليل

الثكل (1): إحداثيات الشريحة المستخدمة في التحليل ودرجة حريتها.

$U^{p}=\sum_{m=1}^{n}\left[C_{u}\right]\left\{U^{p}{ }_{m}\right\} \sin \frac{m \pi \theta}{\alpha}$

$U^{p}=\sum_{m=1}^{n}\left[C_{8}, C_{9}, C_{10}, C_{11}\right]\left\{U^{p}{ }_{m}\right\} \sin \frac{m \pi \theta}{\alpha}$

$V^{p}=\sum_{m=1}^{n}\left[C_{V}\right]\left\{V^{p}{ }_{m}\right\} \cos \frac{m \pi \theta}{\alpha}$

$V^{p}=\sum_{m=1}^{n}\left[C_{8}, C_{9}, C_{10}, C_{11}\right]\left\{V^{p}{ }_{m}\right\} \cos \frac{m \pi \theta}{\alpha}$

$\left[C_{u}\right]=\left[C_{V}\right]$

$C_{8}=1-5 S+8 S^{2}-4 S^{3}$

$C_{9}=4 S-4 S^{2}$

$C_{10}=b\left(-2 S+6 S^{2}-4 S^{3}\right)$

$C_{11}=S-4 S^{2}+4 S^{3}$

إن درجات حرية الحركة للأفعال ضمن المستوي لمركبات الإز احة ( 


$$
\begin{aligned}
& \left\{\mathbf{U}^{p}{ }_{m}\right\}=\left[\mathbf{U}_{i m}, \mathbf{U}_{c m},\left(\frac{\partial \boldsymbol{U}}{\partial \boldsymbol{X}}\right)_{c m}, \mathbf{U}_{j m}\right]^{T} \\
& \left\{\mathbf{V}^{p_{m}}\right\}=\left[\mathbf{V}_{i m}, \mathbf{V}_{c m},\left(\frac{\partial \boldsymbol{V}}{\partial \boldsymbol{Y}}\right)_{c m}, \mathbf{V}_{j m}\right]^{T}
\end{aligned}
$$

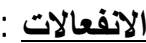

إن" المعادلات الني تثنل العلاقات بين الإزاحة والانفعال للثريحة المخروطية اثثقت من قبل (Novozhilov)

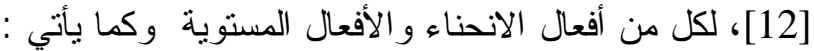

$$
\left\{\begin{array}{c}
\varepsilon_{x} \\
\varepsilon_{\theta} \\
2 \varepsilon_{x \theta} \\
X_{x} \\
X_{\theta} \\
2 X_{x \theta}
\end{array}\right\}=\left\{\begin{array}{c}
\frac{\partial U}{\partial X} \\
\frac{1}{r} \frac{\partial V}{\partial \theta}+\frac{W \cos \phi+U \sin \phi}{r} \\
\frac{1}{r} \frac{\partial U}{\partial \theta}+\frac{\partial V}{\partial X}-\frac{V \sin \phi}{r} \\
-\frac{\partial^{2} W}{\partial X^{2}} \\
-\frac{1}{r^{2}} \frac{\partial^{2} W}{\partial \theta^{2}}+\frac{\cos \phi}{r^{2}} \frac{\partial V}{\partial \theta}-\frac{\sin \phi}{r} \frac{\partial W}{\partial X} \\
2\left(-\frac{1}{r} \frac{\partial^{2} W}{\partial X \partial \theta}+\frac{\sin \phi}{r^{2}} \frac{\partial W}{\partial \theta}+\frac{\cos \phi}{r} \frac{\partial V}{\partial X}-\frac{\sin \phi \cos \phi}{r^{2}} V\right)
\end{array}\right\}
$$
ويمكن تمثيل الانفعال بصيغة مبسطة وكالاتي:

$\{\varepsilon\}=\left[\varepsilon_{x}, \varepsilon_{\theta}, 2 \varepsilon_{x \theta}, \mathbf{X}_{x}, \mathbf{X}_{\theta}, 2 \mathbf{X}_{x \theta}\right]^{T}$

$\{\varepsilon\}=\left[\mathbf{B}_{m}\right]\left\{\delta_{m}\right\}$

$$
\left\{\delta_{m}\right\}=\left[\boldsymbol{U}_{i m}, \boldsymbol{V}_{i m}, \boldsymbol{W}_{i m}, \boldsymbol{\theta}_{i m}, \boldsymbol{U}_{c m}, \boldsymbol{V}_{c m},\left(\frac{\partial \boldsymbol{U}}{\partial \boldsymbol{X}}\right)_{c m},\left(\frac{\partial \boldsymbol{V}}{\partial \boldsymbol{Y}}\right)_{c m}, \boldsymbol{W}_{c m}, \boldsymbol{\theta}_{c m}, \gamma_{c m}, \boldsymbol{U}_{j m}, \boldsymbol{V}_{j m}, \boldsymbol{W}_{j m}, \boldsymbol{\theta}_{j m}\right]^{T}
$$

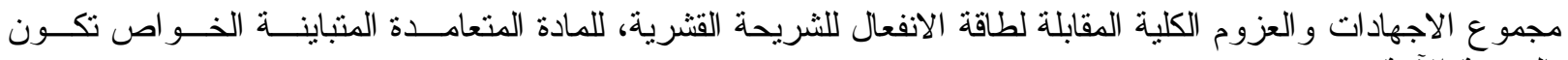

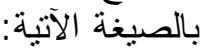

$$
\left\{\sigma_{m}\right\}=\left[\sigma_{x}, \sigma_{\theta}, \sigma_{x \theta}, \mathbf{M}_{x}, \mathbf{M}_{\theta}, \mathbf{M}_{x \theta}\right]^{T}
$$




$$
\left\{\begin{array}{c}
\sigma_{x} \\
\sigma_{\theta} \\
\sigma_{x \theta} \\
\mathbf{M}_{x} \\
\mathbf{M}_{\theta} \\
\mathbf{M}_{x \theta}
\end{array}\right\}=\left[\begin{array}{cccccc}
\mathbf{K}_{x} & \mathbf{K}_{2} & 0 & 0 & 0 & 0 \\
\mathbf{K}_{2} & \mathbf{K}_{\theta} & 0 & 0 & 0 & 0 \\
0 & 0 & \mathbf{K}_{x \theta} & 0 & 0 & 0 \\
0 & 0 & 0 & \mathbf{D}_{x} & \mathbf{D}_{2} & 0 \\
0 & 0 & 0 & \mathbf{D}_{2} & \mathbf{D}_{\theta} & 0 \\
0 & 0 & 0 & 0 & 0 & \mathbf{D}_{x \theta}
\end{array}\right]\left\{\begin{array}{c}
\boldsymbol{\varepsilon}_{x} \\
\boldsymbol{\varepsilon}_{\theta} \\
2 \boldsymbol{\varepsilon}_{x \theta} \\
\boldsymbol{X}_{x} \\
\boldsymbol{X}_{\theta} \\
2 \boldsymbol{X}_{x \theta}
\end{array}\right\}
$$

يمكن تمثيل المعادلة (17) بصيغة مبسطة بالثكل الآتي:

$\left\{\sigma_{m}\right\}=\sum_{m=1}^{n}[\mathbf{D}]\left[\mathbf{B}_{m}\right]\left\{\delta_{m}\right\}$

$$
\begin{aligned}
& \mathbf{D}_{x}=\frac{\mathbf{E}_{x} h^{3}}{12\left(1-v_{x} v_{\theta}\right)}, \quad \mathbf{D}_{2}=v_{\theta} D_{x}, \quad \mathbf{D}_{x \theta}=\frac{\mathbf{G}_{x \theta} h^{3}}{12}, \quad \mathbf{D}_{\theta}=\frac{\mathbf{E}_{\theta} h^{3}}{12\left(1-v_{x} v_{\theta}\right)} \\
& K_{x}=\frac{\mathbf{E}_{x} h}{1-v_{x} v_{\theta}}, K_{2}=v_{\theta} K_{x}, K_{x \theta}=G_{x \theta} h, \quad K_{\theta}=\frac{E_{\theta} h}{1-v_{x} v_{\theta}}
\end{aligned}
$$

الطاقة الكامنة الكلية للشريحة القشرية المخروطية المعرضة إلى الى حمل (q) هي مجموع طاقة الانفعال الداخلية

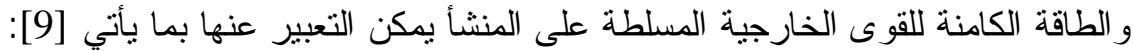

$$
U_{t}=\frac{1}{2} \int_{A}\{\varepsilon\}^{T}\{\sigma\} d A-\int_{A q} q\{\delta\}^{T} d A
$$

$=\frac{1}{2} \sum_{n=1}^{\infty} \sum_{m=1}^{\infty}\left\{\delta_{m}\right\}^{T} \int_{0}^{\alpha} \int_{r_{i}}^{r_{j}}\left[B_{n}\right]^{T}\left[D \mathbf{I} B_{m}\right]\left\{\delta_{m}\right\} r d r d \theta-\sum_{m=1}^{\infty} \int_{A q} q\left\{\delta_{m}\right\}^{T}[C] r d r d \theta$

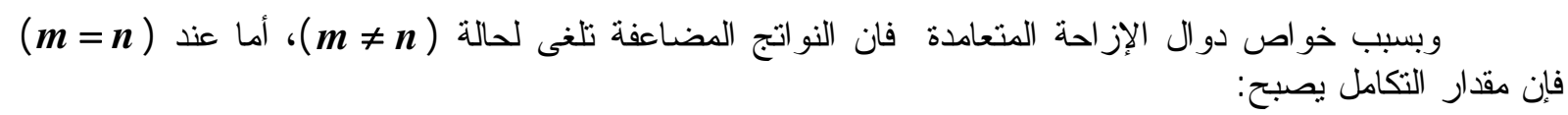

$$
\int_{0}^{\alpha} \sin ^{2} \frac{m \pi \theta}{\alpha} d \theta=\int_{0}^{\alpha} \cos ^{2} \frac{m \pi \theta}{\alpha} d \theta=\frac{\alpha}{2}
$$

بتعويض المعادلة (21) في المعادلة (20) وباختصار التعابير فيما يتعلق بسعة التشوهات يمكن التوصل إلى ما

$\sum_{m=1}^{n} \frac{\alpha}{2} \int_{r i}^{r j}\left[\mathbf{B}_{n}\right]^{T}[\mathbf{D}]\left[\mathbf{B}_{m}\right]\left\{\delta_{m}\right\} r d r=\sum_{m=1}^{n} \int_{A q} q[\mathbf{C}] r d r d \theta$

وتكون مصفوفة الجساءة للشريحة بحجم ( 15×15)، للعدد التو افقي $m$ من المعادلة(22) تمنل بالثكل

$$
\begin{gathered}
{[K]=\frac{\alpha}{2} \int_{r i}^{r j}\left[B_{m}\right]_{15 \times 6}^{T}[\mathbf{D}]_{6 \times 6}\left[\mathbf{B}_{m}\right]_{6 \times 15} r d r} \\
\{\boldsymbol{F}\}=\int_{A q} q[C] r d r d \theta
\end{gathered}
$$


اشتقاق مصفوفة الكتلة المتجانسة

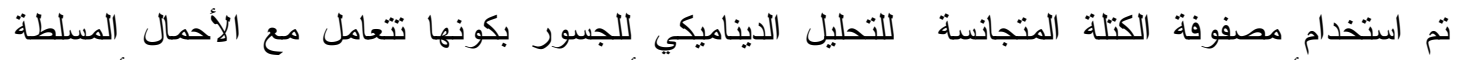

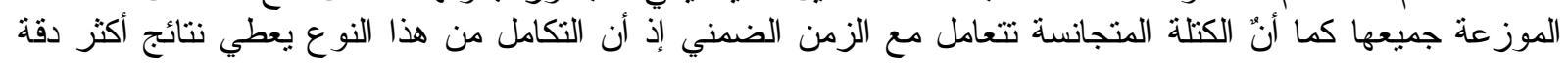

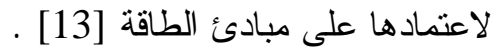

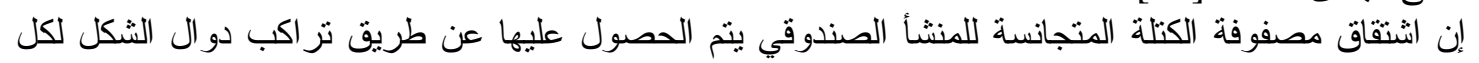

من الأفعال الانحنائية والأفعال المستوية، فالإز احة الافتر اضية لأي شريحة قثترية يتم تمثليها بالصيغة الآتية [14]: $\{f\}=\sum_{m=1}^{n}[N]\left\{\delta_{m}\right\}$

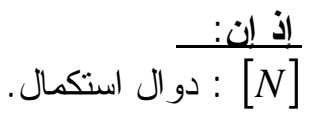

$$
\begin{aligned}
& \text { 吕 : : الإزاحة الافتر اضية للشريحة المخروطية. }
\end{aligned}
$$

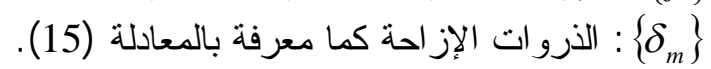

ويعبر عن المشتقة الثانية لمركبات الخطوط العقدية التي تمثل متجه التعجيل بالصيغة الآتية:

$$
\{\ddot{f}\}=\sum_{m=1}^{n}[N]\left\{\ddot{\delta}_{m}\right\}
$$

$\{\bar{q}\}=\rho\{\ddot{f}\}$

ويمكن التعبير عن قوى القصور الذاني للثريحة القترية كما في المعادلة الآتية:

年

إن مقدار القوى العقدية (Nodal Forces) المكافئة لقوى القصور الذاتي للثريحة القشرية تحسب كما في

$\{\boldsymbol{F}\}=\int_{v}[N]^{T}\{\bar{q}\} d V$

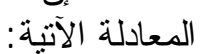

وطبقا لمعادلة تو ازن الحركة للمنثأ فإن القوى العقدية تعرف كما يأتي:

$\{\boldsymbol{F}\}=\left[\boldsymbol{M}_{c}\right]\left\{\ddot{\boldsymbol{\delta}}_{m}\right\}$

$\rho \int_{v}[N]^{T}[N] d V\left\{\ddot{\delta}_{m}\right\}=\left[M_{c}\right]\left\{\ddot{\delta}_{m}\right\}$

وبتعويض المعادلة (29) في المعادلة (28) ينتج الآتي:

$\left[M_{c}\right]=\rho h \int_{A}[N]^{T}[N] d($ area $)$

إن المعادلة النهائية لمصفوفة الكتلة المتجانسة تكون كما يأتي:

وباستخدام الثريحة القشرية المخروطية ذات النسق العالي (دالة الثكل بالاتجاه العمودي ودالة الثكل بالاتجاه

و بك الثريحة القشرية.

$$
\begin{aligned}
& {[N]=[C] \sin \frac{m \pi \theta}{\alpha}} \\
& \text { المسنوي للانحناء)، فإن مصفوفة [N] } \\
& {\left[M_{c}\right]=\sum_{n=1}^{\infty} \sum_{m=1}^{\infty} \int_{r i}^{r j}[\mathrm{C}][\mathrm{C}] r d r \int_{0}^{\alpha} \rho h \sin ^{2} k m d \theta} \\
& k m=\frac{m \pi \theta}{\alpha}
\end{aligned}
$$


$\left[\mathbf{M}_{c}\right]=\frac{\rho h \alpha}{2} \int_{r i}^{r j}[\mathbf{C}]_{15 \times 3}^{T}[\mathbf{C}]_{3 \times 15} r d r$

ونم الحصول على مصفوفة الكتلة المتجانسة بالحل الرياضي لمعادلات من الدرجة السادسة و الثالثة، ثم إجر اء إثاء

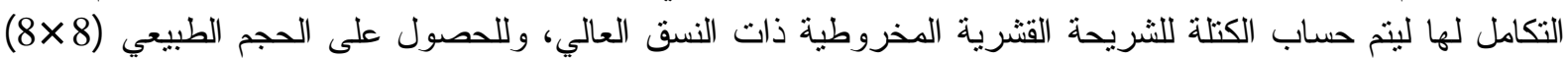
للنسق الو اطئ لمصفوفة الكتلة ذات النسق العالي يتم عمل تكثيف لمصفوفة الكتلة لحذف درجات الحرية الإضافية للخط

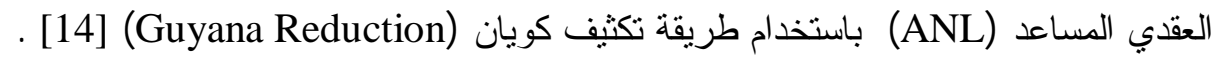

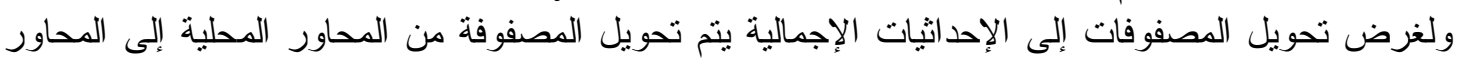

$$
\left[\bar{M}_{c}\right]=[T]\left[M_{c}\right][T]^{T}
$$

الإجمالية بموجب العلاقة الآتية:

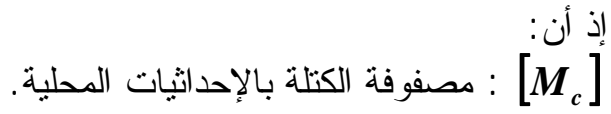

$$
\begin{aligned}
& \text { ] : مصفوفة الكتلة بالإحداثيات الإجمالية. }
\end{aligned}
$$

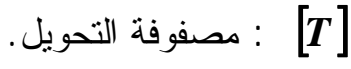

$$
\begin{aligned}
& \text { طريقة تكثيف كويان }
\end{aligned}
$$

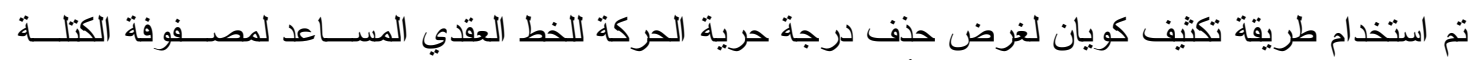

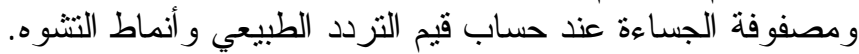

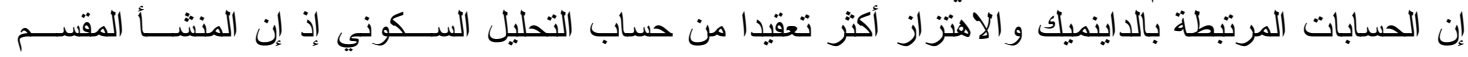

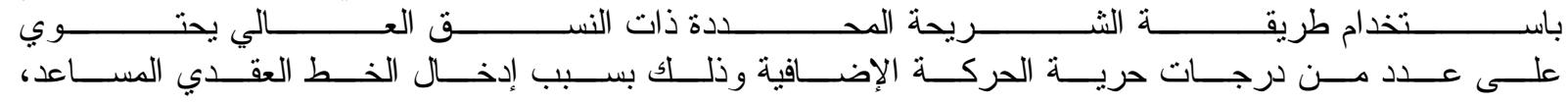

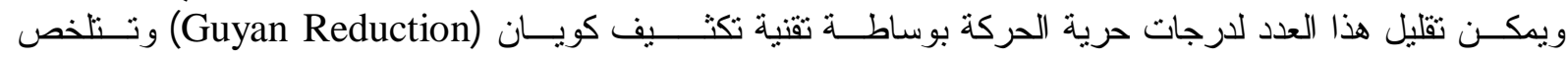
$\left(\left[\begin{array}{ll}K_{o o} & K_{o i} \\ K^{T}{ }_{o i} & K_{i i}\end{array}\right]-\omega^{2}\left[\begin{array}{ll}M_{o o} & M_{o i} \\ M^{T}{ }_{o i} & M_{i i}\end{array}\right]\right)\left\{\begin{array}{l}\delta_{o} \\ \delta_{i}\end{array}\right\}=0$

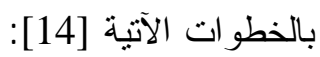

: Koo

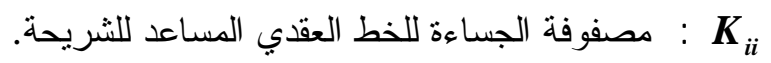
: Moo : : : Mصفوفة الكتلة للخط العقدي المساعد للشريحة. : : $\delta_{o}$ إن : متجه الإز احة عند الخط العقدي المساعد. إن مقدار الإز احة للخط العقدي المساعد ينم حسابها بالاعنماد على المعادلة الآتية:

$\left\{\delta_{i}\right\}_{i \times 1}=-\left[K_{i i}\right]_{i i}^{-1}\left[K_{o i}\right]_{i i}^{T}\left\{\delta_{o}\right\}_{o \times 1}$

وقد تم إهمال قيمة مصفوفة الكتلة للشريحة القشرية في المعادلة (37) وذللك لان قيمتها صــيرة قياســا إلـى مقــار مصفوفة الجساءة.

$\left\{\begin{array}{l}\delta_{o} \\ \delta_{i}\end{array}\right\}_{z \times 1}=\left[T_{n}\right]_{z \times o}\left\{\delta_{o}\right\}_{o \times 1}$

o 0 : : عدد درجات حرية الحركة للخط العقدي المساعد. : i

مجمو عرجات حرية الحركة (o+i) : z ] $]$ ] : مصفوفة التدوير و التي تحسب بالثكل الآتي: 
وبتعويض المعادلة (39) في المعادلة (36) وضربها بالمصفوفة

$\left[\boldsymbol{K}_{r}\right]_{o \times 0}=\left[\boldsymbol{T}_{n}\right]^{T}[\boldsymbol{K}]_{z \times z}\left[\boldsymbol{T}_{n}\right]$

$\left[\boldsymbol{M}_{r}\right]_{o \times o}=\left[\boldsymbol{T}_{n}\right]^{T}[\boldsymbol{M}]_{z \times z}\left[\boldsymbol{T}_{n}\right]$

وبذللك تصبح مصفوفة الجساءة و الكتلة النهائية بحجم (8 ×8) بدلا من (15 ×15).

الأمثلة التطبيقية

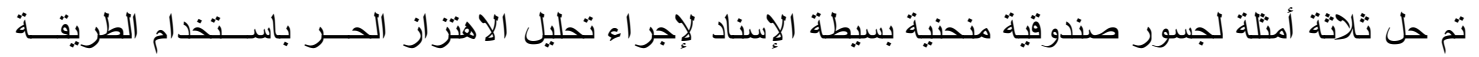

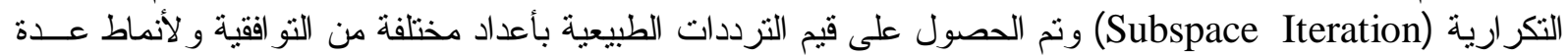

للتنشوه.

المثال الأول: الاهتزاز الحر لجسر صندوقي منحن مؤلف من خليتين (15) -1) (Bridge)

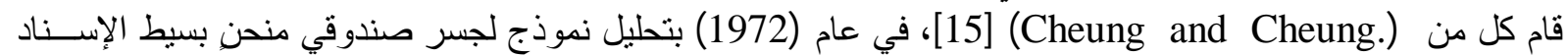

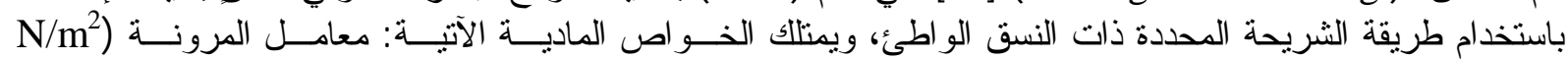

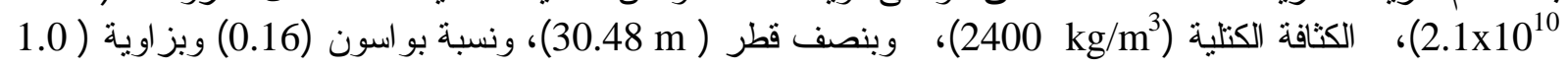

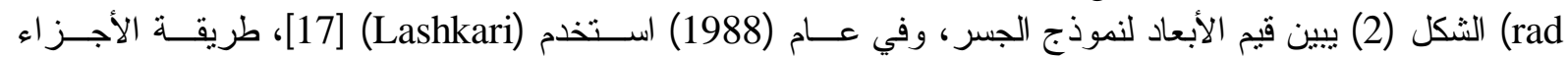

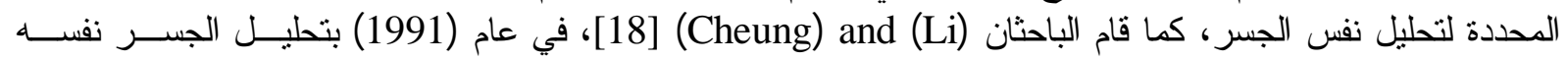

بطريقة (Spline Finite Strip).

وقد نم في الدر اسة الحالية تقسيم المقطع الصندوقي إلى (13) شريحة محددة ذات النسق العالي كما مبين فـي

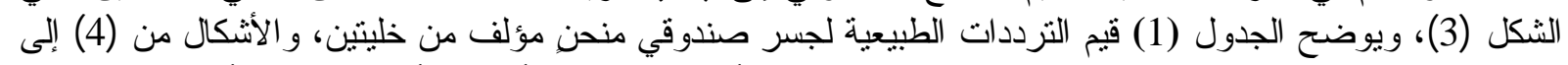

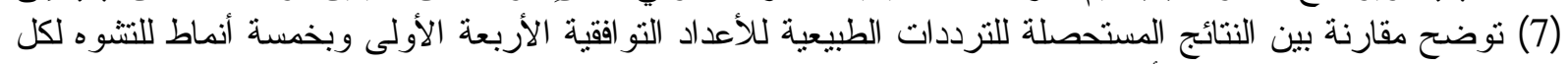

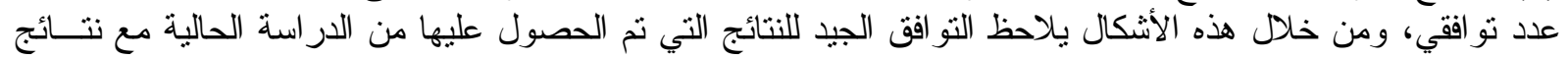

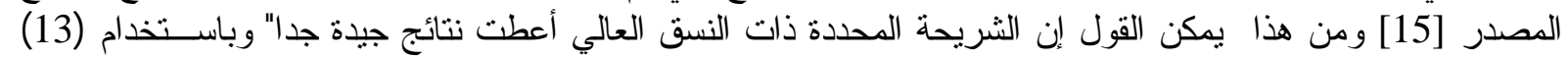

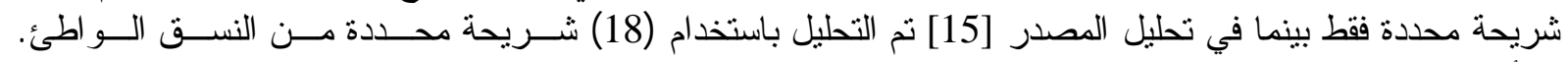

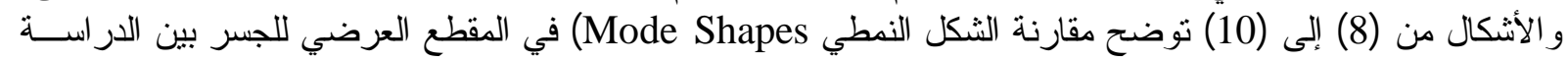

0.203

الحالية و الدر اسة السابقة [15] ولقيم مختلفة من الترددات الطبيعية.
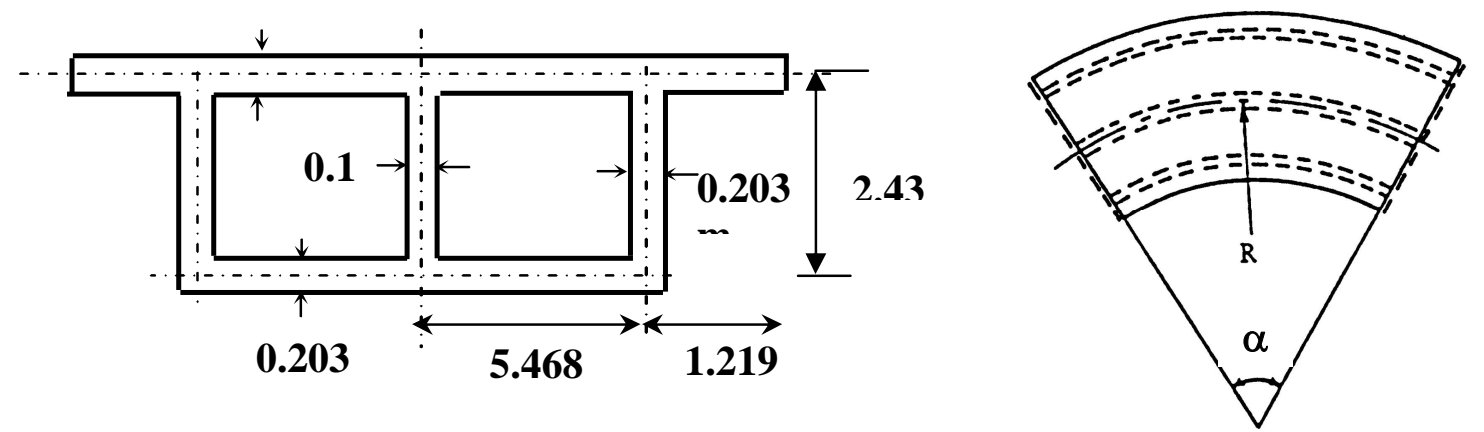

(الشكل(2): الأبعاد للجسر الصندوقي المنحني (Bridge-1)

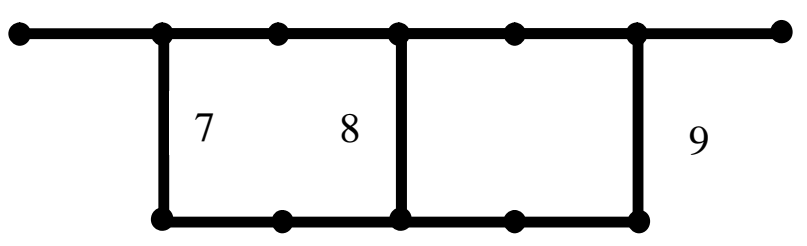

$10 \quad 11 \quad 12 \quad 13$

الثكل(3): تقسيم المقطع الصندوقي (Bridge-1) إلى شرائح 
عبد الرزاق : تحليل الاهتزاز الحر للجسور الصندوقية المنحنية بطريقة الثريحة المحدة ذات النسق العالي

الجدول (1) يوضح قيم الترددات الطبيعية لجسر صندوقي منحن مؤلف من خليتين (Bridge-1)

\begin{tabular}{|c|c|c|c|c|c|}
\hline $\begin{array}{c}\text { No. of } \\
\text { Harmoninc }\end{array}$ & $\begin{array}{c}\text { Circuler } \\
\text { Frequency } \\
\text { (Rad./sec.) } \times \mathbf{1 0}^{-3}\end{array}$ & $\begin{array}{l}\text { Cheung and } \\
\text { Cheung [15] }\end{array}$ & $\begin{array}{c}\text { Lashkari } \\
{[17]}\end{array}$ & $\begin{array}{c}\text { Cheung } \\
\text { and } \operatorname{Li}[18]\end{array}$ & $\begin{array}{l}\text { Present } \\
\text { Study }\end{array}$ \\
\hline \multirow[t]{5}{*}{$\mathrm{m}=1$} & $\omega_{1}$ & 2.623 & 2.616 & 2.62 & 2.64 \\
\hline & $\omega_{2}$ & 4.252 & 4.251 & 4.249 & 4.293 \\
\hline & $\omega_{3}$ & 8.064 & 8.037 & 8.066 & 8.204 \\
\hline & $\omega_{4}$ & 8.501 & 8.471 & 8.501 & 8.589 \\
\hline & $\omega_{5}$ & 8.554 & 8.49 & 8.553 & 8.65 \\
\hline \multirow[t]{5}{*}{$\mathrm{m}=2$} & $\overline{\omega_{1}}$ & 8.002 & 8.002 & 7.945 & 8.03 \\
\hline & $\omega_{2}$ & 8.605 & 8.603 & 8.605 & 8.763 \\
\hline & $\omega_{3}$ & 9.107 & 9.017 & 9.107 & 9.2311 \\
\hline & $\omega_{4}$ & 11.427 & 11.43 & 11.427 & 11.645 \\
\hline & $\omega_{5}$ & 13.644 & & & 13.65 \\
\hline \multirow[t]{5}{*}{$\mathrm{m}=3$} & $\omega_{1}$ & 9.436 & 9.436 & 9.436 & 9.625 \\
\hline & $\omega_{2}$ & 10.257 & 10.257 & 10.257 & 10.432 \\
\hline & $\omega_{3}$ & 11.821 & & & 12.011 \\
\hline & $\omega_{4}$ & 12.246 & & & 12.509 \\
\hline & $\omega_{5}$ & 20.046 & & & 20.34 \\
\hline \multirow[t]{5}{*}{$m=4$} & $\omega_{1}$ & 10.608 & 10.608 & 10.608 & 10.83 \\
\hline & $\omega_{2}$ & 11.556 & & & 11.78 \\
\hline & $\omega_{3}$ & 13.49 & & & 13.67 \\
\hline & $\omega_{4}$ & 13.523 & & & 13.8 \\
\hline & $\omega_{5}$ & 25.243 & & & 25.54 \\
\hline
\end{tabular}

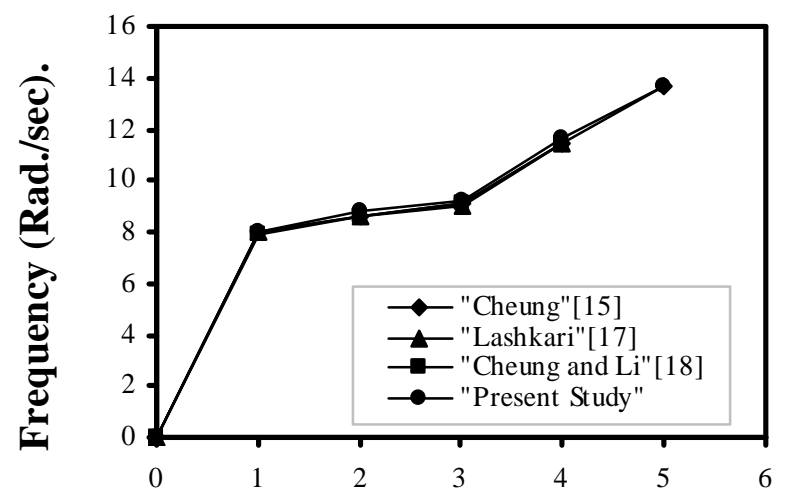

Number of Mode

الثكل (5): الترددات الطبيعية للجسر الصندوقي (Bridge-1)

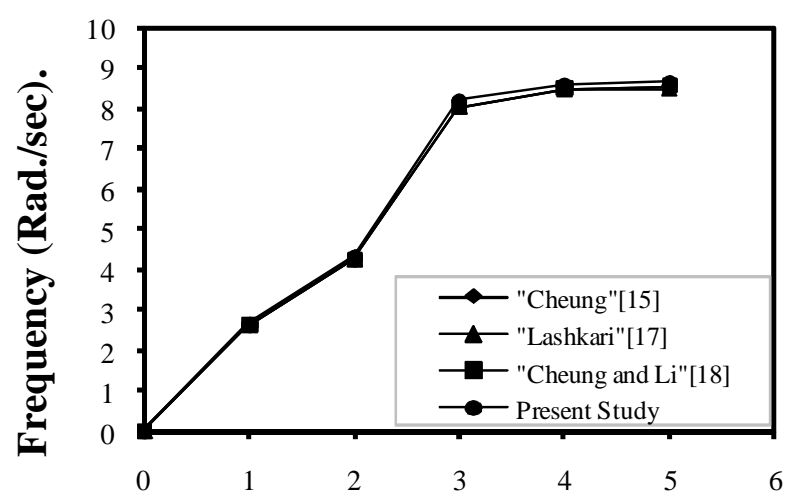

Number of Mode

الثكل (4): الترددات الطبيعية للجسر الصندوقي (Bridge-1) 


\begin{tabular}{llll} 
Al-Rafidain Engineering & Vol.17 & No.4 & August 2009 \\
\hline
\end{tabular}

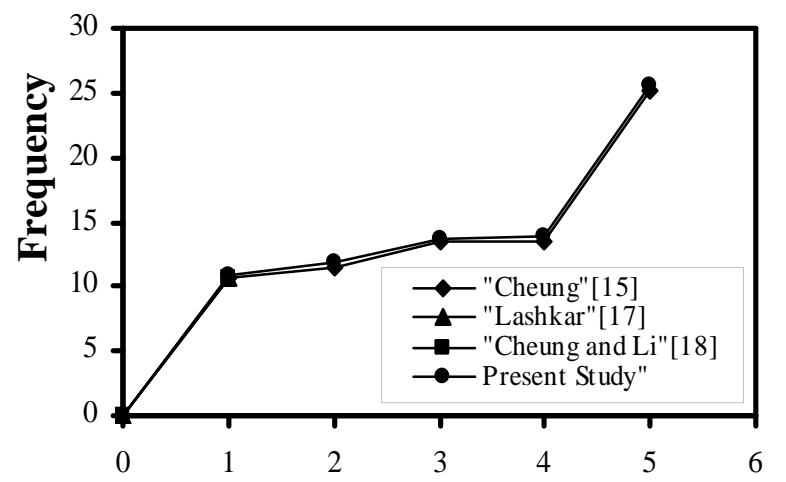

Number of Mode

الثكل (7): الترددات الطبيعية للجسر الصندوقي (Bridge-1)

Present Study

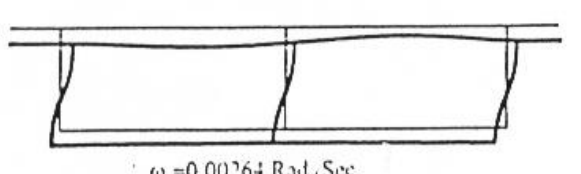

$\omega=0.0026+$ Rad. Sec

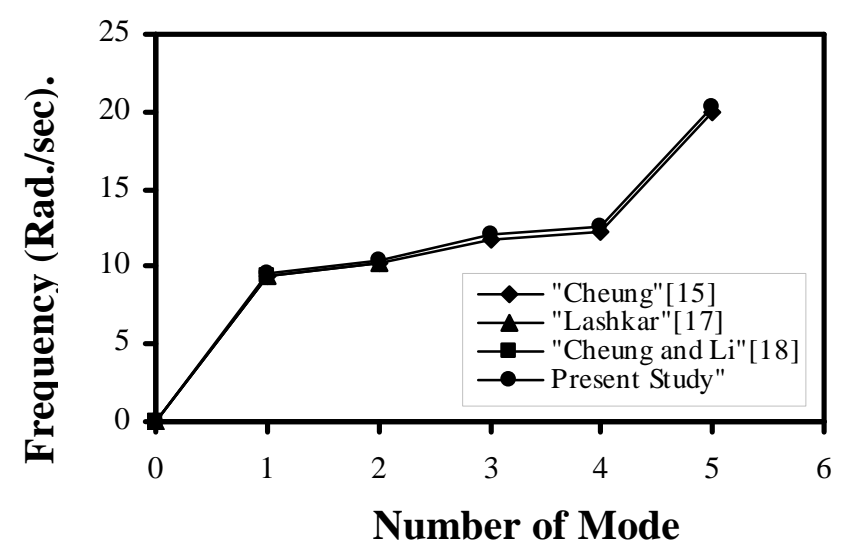

الثكل (6): الترددات الطبيعة للجسر الصندوقي (Bridge-1)

Cheung [15]

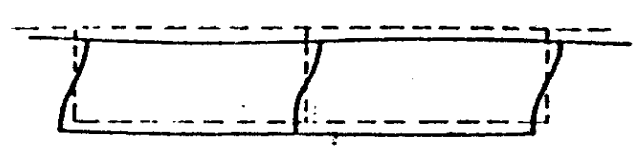

الثكل (8): نمط الثكل الأول للجسر الصندوقي (1- Bridge) للعدد التوافقي الأول

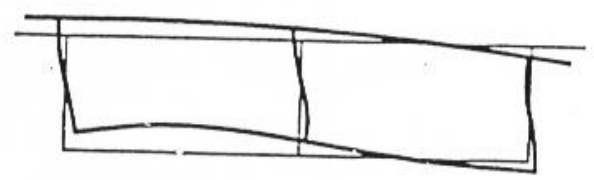

$\omega=0.00+29$ Rad $/$ Sec

الثكل (9): نمط الثكل الثاني للجسر الصندوقي (Bridge -1) للعدد التو افقي الثاني
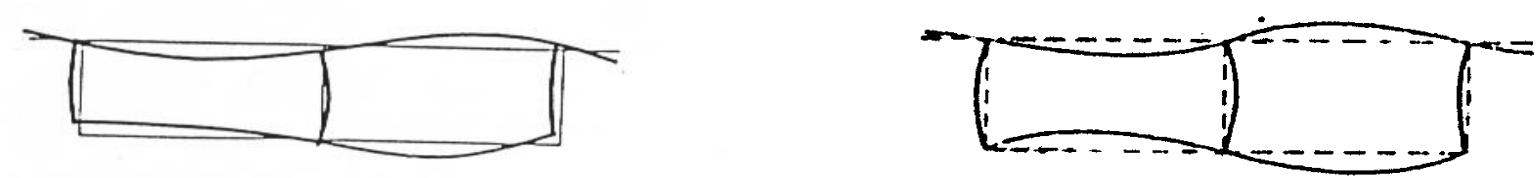

(1) $=0.00962$ Rad. Ste
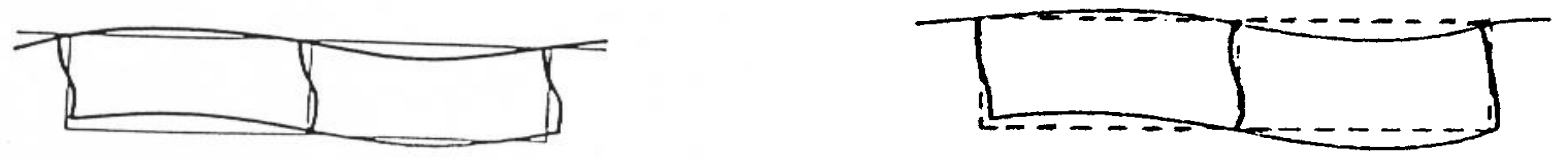

(s) $=0,0 ; 04$ R.al Sis

الثكل (10): نمط الثكل الأول و الثاني للجسر الصندوقي (Bridge -1) للعدد التو افقي الثالث 


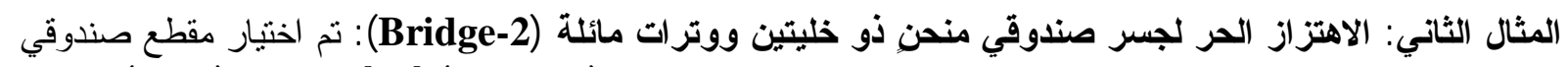

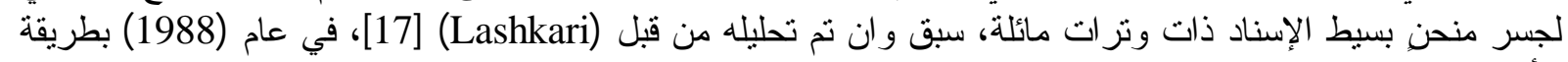
الأجز اء المحددة.

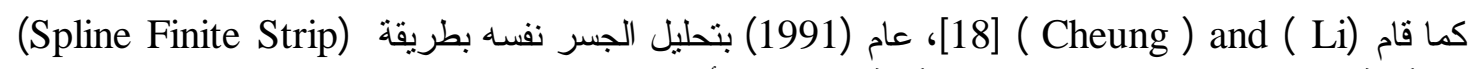

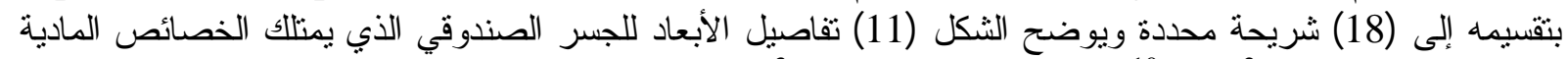

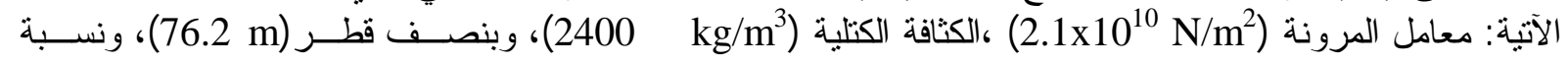

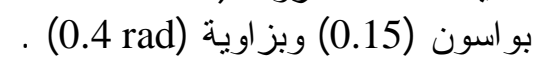

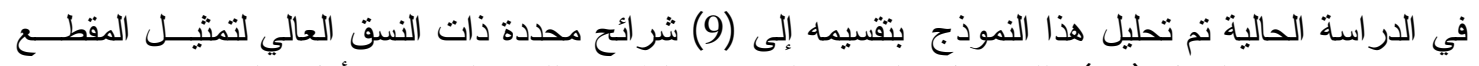

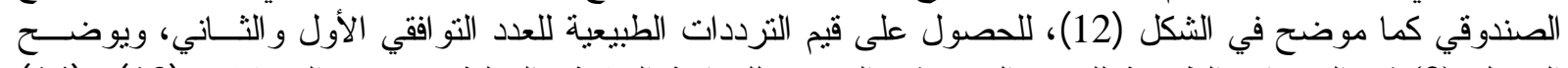

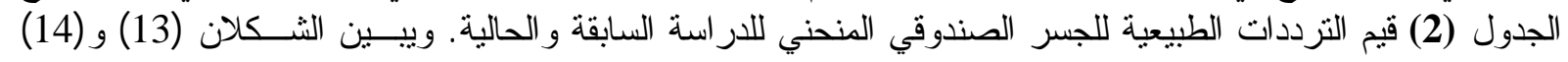

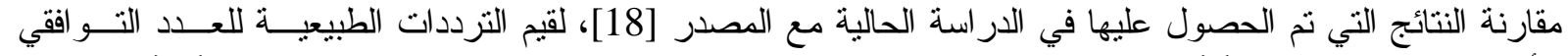

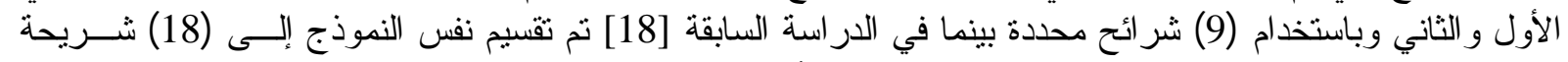

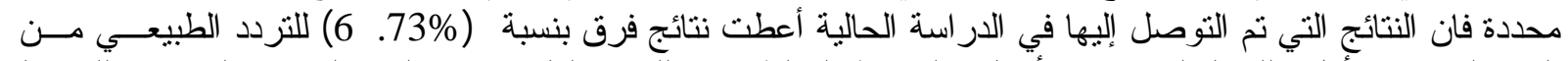

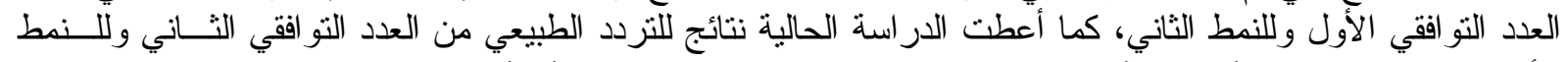

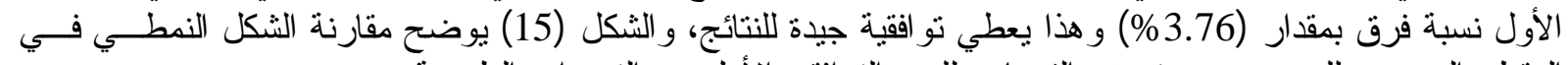

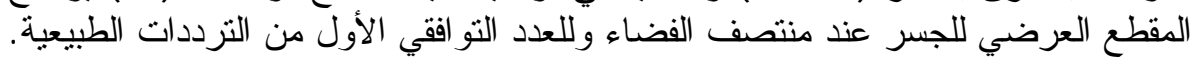
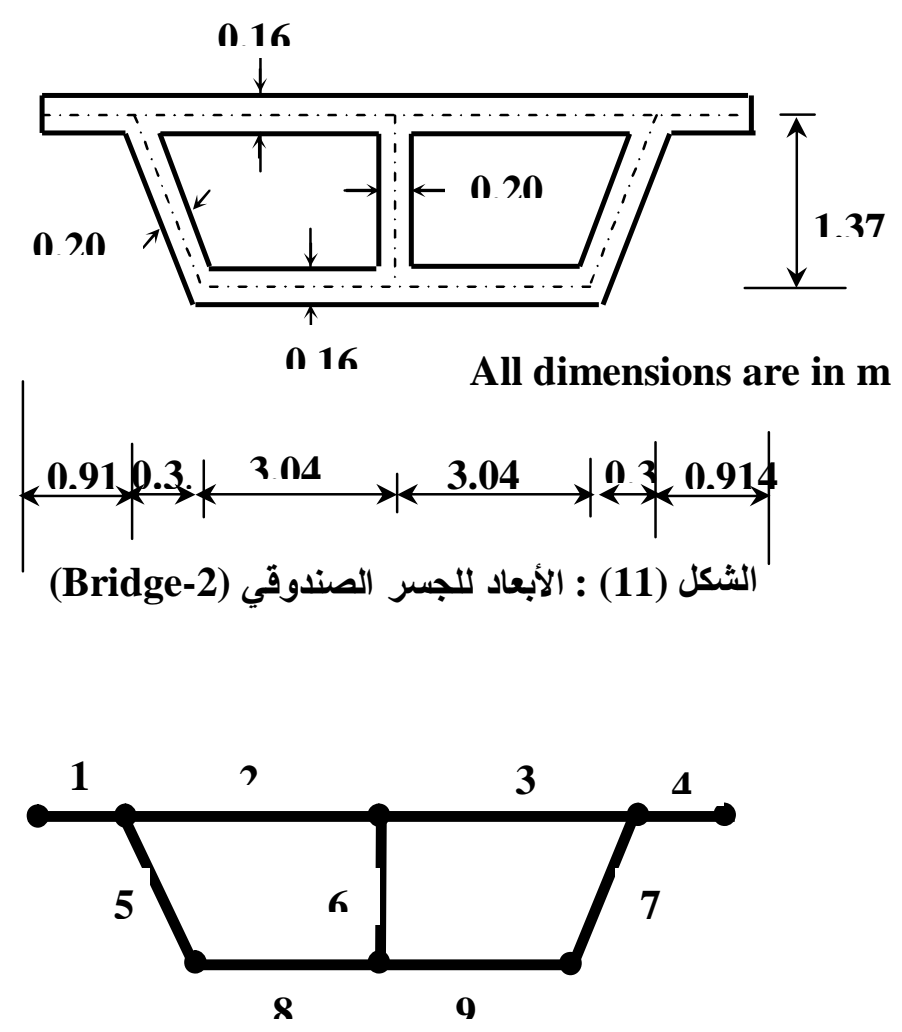

الشكل (12) : تقسيم مقطع الجسر الصندوقي (Bridge-2) 


\begin{tabular}{llll} 
Al-Rafidain Engineering & Vol.17 & No.4 & August 2009 \\
\hline
\end{tabular}

الجدول (2) يوضح قيم الترددات الطبيعية لجسر صندوقي منحني مؤلف من خليتين (Bridge-2)

\begin{tabular}{|l|c|c|c|c|}
\hline $\begin{array}{r}\text { No. of } \\
\text { Harmoninc }\end{array}$ & $\begin{array}{c}\text { Circuler } \\
\text { Frequency } \\
\text { (Rad./sec.) } \times 10^{-3}\end{array}$ & $\begin{array}{c}\text { Lashkari } \\
{[\mathbf{1 7}]}\end{array}$ & $\begin{array}{c}\text { Cheung } \\
\text { and } \\
\text { Li[18] }\end{array}$ & $\begin{array}{c}\text { Present } \\
\text { Study }\end{array}$ \\
\hline $\mathrm{m}=1$ & $\omega_{1}$ & 18.307 & 18.405 & 18.468 \\
\cline { 2 - 5 } & $\omega_{2}$ & 67.198 & 62.343 & 62.67 \\
\cline { 2 - 5 } & $\omega_{3}$ & 67.565 & 67.831 & 68.34 \\
\cline { 2 - 5 } & $\omega_{4}$ & 117.22 & 116.58 & 117.38 \\
\hline $\mathrm{m}=2$ & $\omega_{1}$ & 72.815 & 69.242 & 70.076 \\
\cline { 2 - 5 } & $\omega_{2}$ & 107.08 & 101.68 & 103.26 \\
\cline { 2 - 5 } & $\omega_{3}$ & & & 174.03 \\
\cline { 2 - 5 } & $\omega_{4}$ & & & 210.85 \\
\hline
\end{tabular}

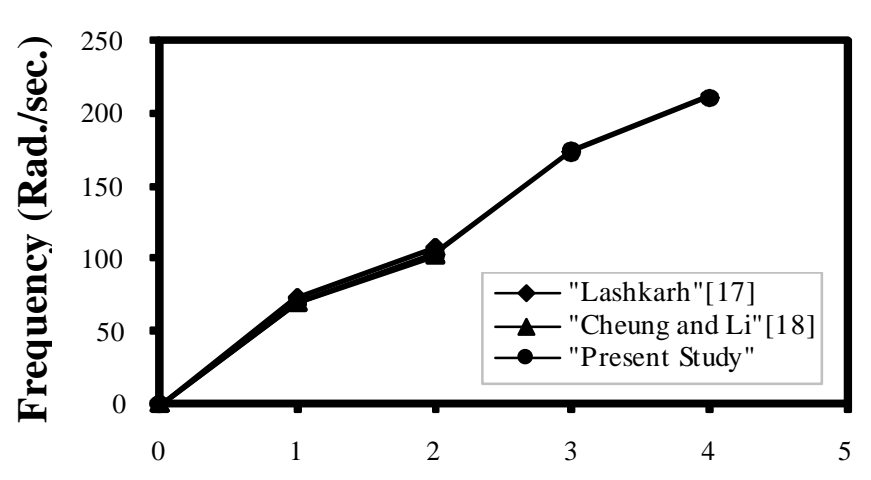

Number of Mode

الثنكل (14): الترددات الطبيعية للجنروير الصندوقي(Bridge-2) للعدد التو اقفتي الثاني

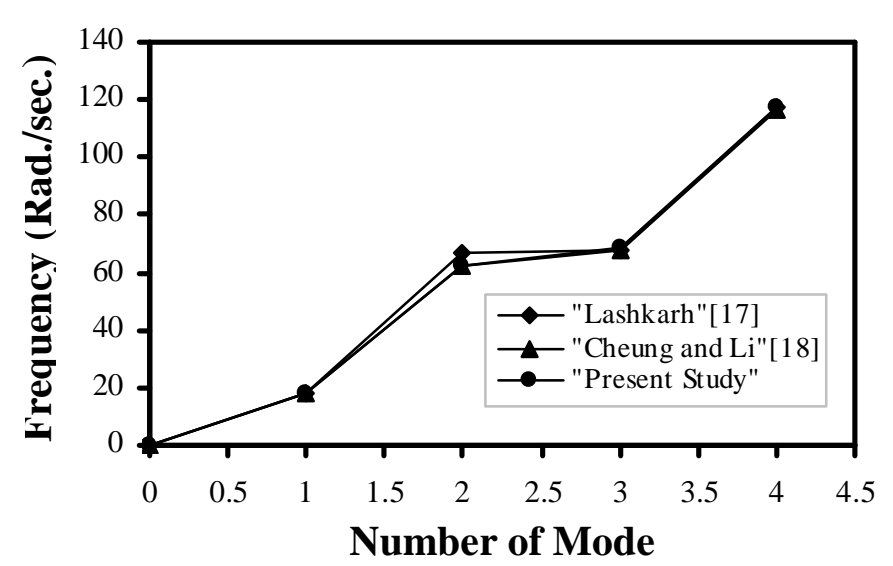

Number of Mode

الثكل (13): الترددات الطبيعية للجسر الصندوقي(Bridge-2) للعدد التو افققي الأول
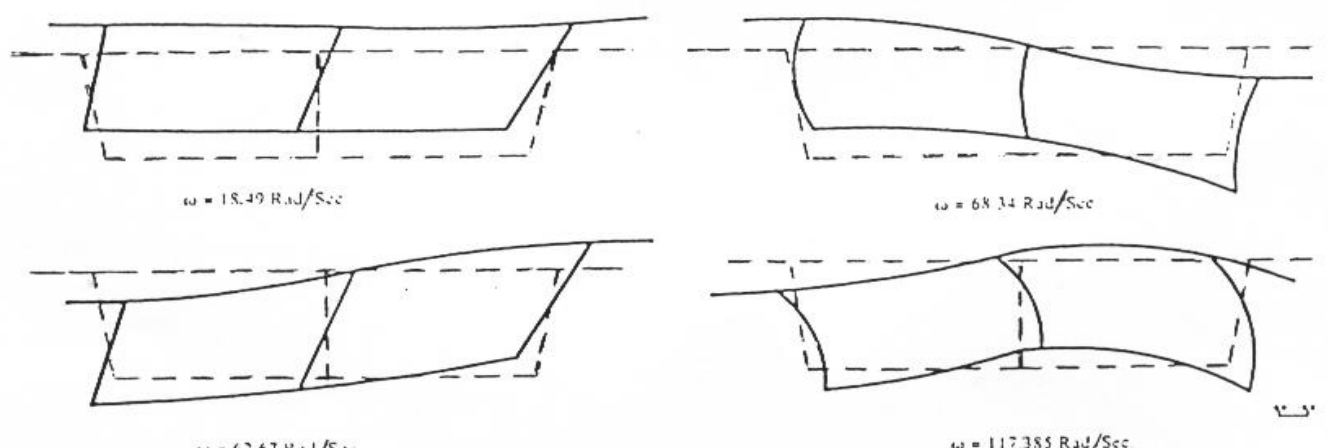

الثكل (15): نمط الثكل للجسر الصندوقي (Bridge -2) للعدد التو افقي الأول 
المثال الثالث: الاهتزاز الحر لجسر صندوقي منحن يتألف من خلية واحدة (Bridge-3):

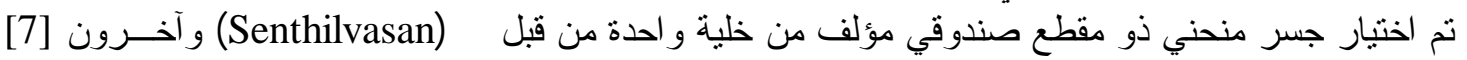

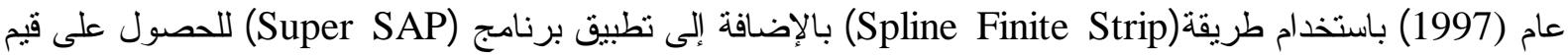

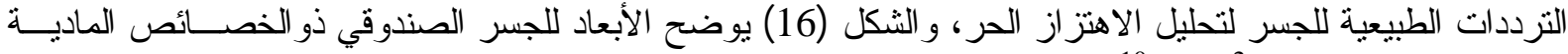
الآتية: معامل المرونة (2.1x10 N/m

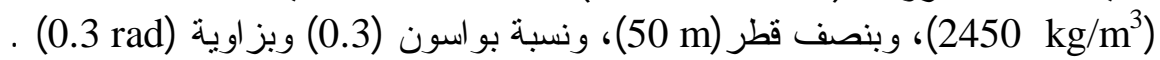
في الدر اسة الحالية تم تحليل الجسر وذلك بثقسيم المقطع الصندوفي إلى ثمان شرائح محددة ذات النسق العالي و الموضحة بالثنكل (17). يوضح الجدول (3) النو افق الجيد في قيم الترددات الطبيعية للدر اسة الحالية و الدر اســـة الدقدمـة مــن قبــلـ

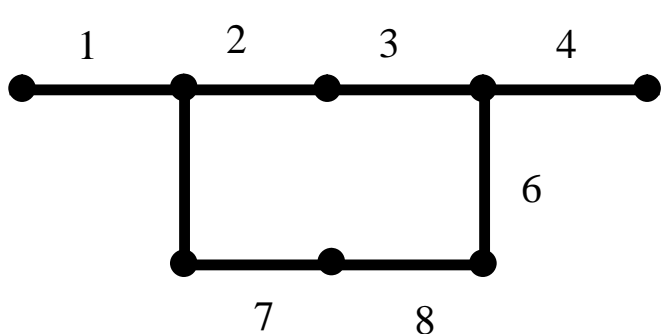

(17) الثكل (Bridge-3) (تقسيم المقطع الصندوقي (17) إلى شر ائح محددة المنام

الجدول (3) يوضح قيم الترددات الطبيعية لجسر صندوقي منحني مؤلف من خلية واحدة (Bridge-3)

\begin{tabular}{|c|c|c|c|c|}
\hline $\mathrm{m}=1$ & $\begin{array}{c}\text { Circuler } \\
\text { Frequency (Hz) }\end{array}$ & $\begin{array}{c}\text { Super } \\
\text { SAP [7] }\end{array}$ & Senthilvasan [7] & $\begin{array}{c}\text { Present } \\
\text { Study }\end{array}$ \\
\cline { 2 - 5 } & $\omega_{1}$ & 3.79 & 3.66 & 3.25 \\
\hline$\omega_{2}$ & 4.11 & 4.07 & 3.69 \\
\hline$\omega_{3}$ & 4.64 & 4.29 & 4.6 \\
\hline$\omega_{4}$ & 4.98 & 5 & 5.1 \\
\hline & $\omega_{5}$ & 5.2 & 5.32 & 5.87 \\
\hline
\end{tabular}

الأستنتاجات

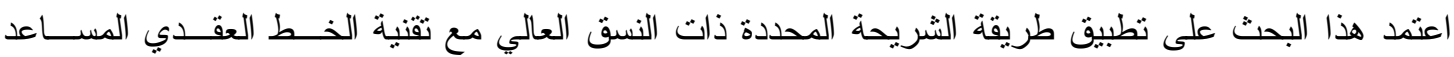

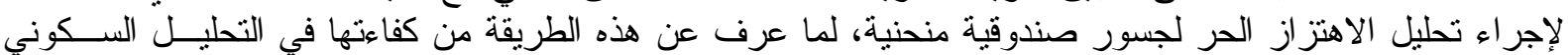
للجسور حسب ما أظهرته البحوث الإن السابقة.

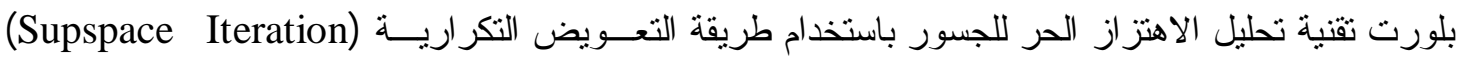

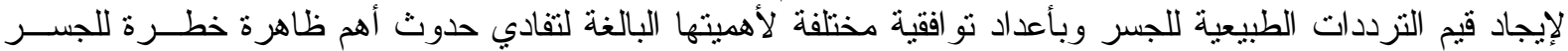

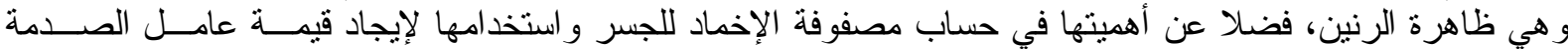
للجسر عند إجراء التحليل الديناميكي. فئل أهني.

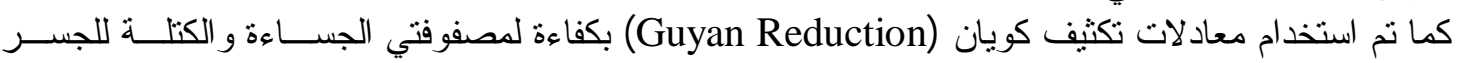

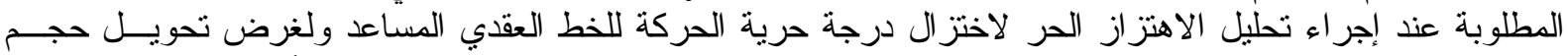

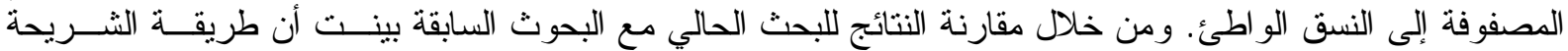

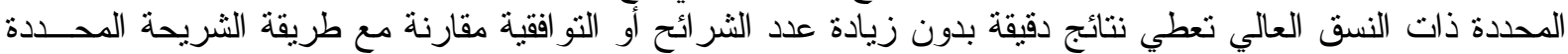

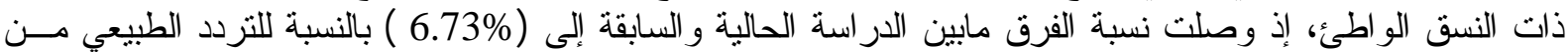

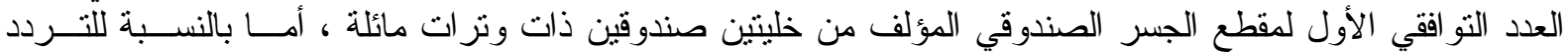

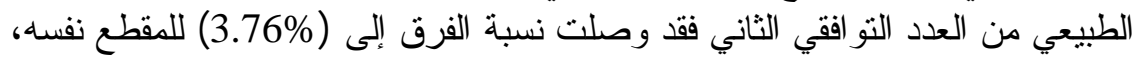
كما نم التوصل على أوطاً تردد بالاعتماد على قيمة النمط الأول للاهتزاز من العدد التو افقي الأول. 
1. Cheung, Y.K., "Orthotropic Right Bridges by The Finite Strip Method", Concrete Bridge Design, ACI, SP-26, 1971, PP.357-378.

2. Humar, J.L. and Kashif, A.M., "Dynamic Response of Bridges under Travelling Loads", Canadian Journal of Civil Engineering, Vol.20, 1993, PP.287-298.

3. Komatsu, S. and Nakai, H., "Study of Free Vibration of Curved Girder Bridge" Transactions of The Japan Society of Civil Engineers, Tokyo, Japan, Vol.136, Dec. 1966.

4. Culver, C.G. and Oestel, D.J., "Natural Frequencies of Multispan Curved Beams", Journal of Sound and Vibration, Vol.10, No.3, 1969, PP. 380-389.

5. Sheikh, A.H. and Mukhopadhyay, M., "Free Vibration Analysis of Stiffned Plates with Arbitrary Planform by the General Spline Finite Strip Method", Journal of Sound and Vibration, Vol.162, No.1, 1993, PP.147-164.

6. Huang, D. and Member, P.E., "Dynamic Analysis of Steel Curved Box Girder Bridges”, Journal of Bridges Engineering, Vol.6, No.6, Dec. 2001, PP.506512.

7. Senthilvasan, J., Brameld, G.H. and Thambiratnam, D.P., "Bridge-Vehicle Interaction in Curved Box Girder Bridges", Micro Computers in Civil Engineering, Vol.12, 1997, PP.171-181

8. Abdul-Razzak, A.A., and Mohammad, A.A., "Dynamic Analysis of Box Girder Bridges Using Higher Order Finite Strip Method", AL-Rafidain Engineering Journal, Vol.10, No.1, 2005, PP.1-74.

9. Abdul-Razzak, A.A, "Finite Strip Analysis of Curved Box Girder", $\boldsymbol{A L}$ Rafidain Engineering Journal, Vol.2, No.2, 1994, PP.122-142

10. Abdul-Razzak, A.A. and Najem, R.M., "Solution of Curved Box Girder Bridges with Intermediate Diaphragms", AL-Rafidain Engineering Journal, Vol.10, No.1, 2002, PP.1-22.

11. Abdul-Razzak, A.A. and Najem, R.M., "Solution of Curved Box Girder Bridges on Flexible Bent", Scientific Journal of Tikrit University, Vol.8, No.3, Sept. 2002, PP.38-54

12. Novozhilov, V.V., "Theory of Thin Shells", Second Edition, Groningen, P. Noordhoff, 1964.

13. Senthilvasan, J., Thambiratnam, D.P. and Brameld, G.H.,"Dynamic Response of A Curved Bridge under Moving Truck Load", Engineering Structures, Vol.24, No.10, 2002, PP.1283-1293.

14. Cook, R.D., "Concepts and Application of Finite Element Analysis", John Wiley and Sons, Second Edition, 1981, PP.537.

15. Cheung, Y.K. and Cheung, M.S., "Free Vibration of Curved and Straight Beam-Slab or Box Girder Bridges", Publications International Association for Bridge and Structural Engineering, 32 -II, 41-52,1972.

16. Cheung, Y.K., "Finite Strip Method in Structural Analysis", Pergamon Press, 1976, PP.232.

17. Lashkari, M., "COSMOS/M User Guide ", Structural Research and Analysis Corporation, 1988.

18. Cheung,Y.K. and Li, W.Y., "Free Vibration Analysis of Longitudinal Arbitrarily Curved Box- Girder Structures by Spline Finite Strip Method", Computational Mechanics Balkema, Rotterdam, 1991, PP.1139-1144.

تم اجراء البحث في كلية الهنسة - جامعة الموصل 